\title{
Introducción de valores transversales en la docencia de Periodismo: el caso de la solidaridad
}

\author{
Carmen del Rocío Monedero Morales \\ Universidad de Málaga \\ roi@uma.es \\ Silvia Olmedo SAlar \\ Universidad de Málaga \\ silviaolmedo@uma.es
}

\begin{abstract}
Resumen:
La entrada en vigor del Espacio Europeo de Educación Superior (EEES) ha supuesto una apuesta formativa por la adquisición de competencias, posibilitando y promoviendo su desempeño profesional por parte del alumnado. Para cumplir con estos objetivos el proceso formativo ha de ir acompañado por praxis que impulsen el desarrollo de valores que nutran el rol de ciudadano, como es el caso de la solidaridad.

El texto que se presenta aborda la experiencia innovadora realizada con estudiantes del Grado en Periodismo (curso 2012/13) así como las claves para que pudiera extrapolarse a otras materias del campo de las Ciencias Sociales.
\end{abstract}

Palabras clave: solidaridad, periodismo, comunicación, competencias

\section{Cross-cutting topic introduction in teaching Journalism: the case of solidarity}

\begin{abstract}
:
The entry into force of the European Higher Education Area (EHEA) has made a commitment to training for the acquisition of skills, enabling and promoting students professional performance. To meet these objectives the training process must be accompanied by practice to foster the development of values that nurture citizen's role, as in the case of solidarity.

The text presented addresses the innovative experience made with students of second course of the degree in Journalism (course 2012/13). Also the keys that it shows could be extrapolated to other subjects in the field of Social Sciences.
\end{abstract}

Key Words: solidarity, journalism, communication, skills

\section{Referencia normalizada:}

Monedero Morales, C.R. y Olmedo Salar, S. (2014): Introducción de valores transversales en la docencia de periodismo: el caso de la solidaridad. Historia y Comunicación Social. Vol. 19. Núm. Especial Enero. Págs. 833-846.

Sumario: 1. Introducción. 2. Hacia una relación entre solidaridad y competencias académicas. 3. La necesidad de dar a conocer a actores alternativos en el sistema mediático. 4. La apuesta solidaria y el Grado en Periodismo. 5. Resultados de la experiencia en el aula. 6. Conclusiones finales 6. Bibliografía. 


\section{Introducción}

Consideramos que la adquisición de competencias del alumnado que posibilite su desempeño profesional debe ir acompañado de praxis que impulsen el desarrollo de valores que nutran su rol de ciudadano. De tal modo que valores como la solidaridad, el ecologismo, la igualdad y la justicia, por citar solo algunos, deben estar presente de manera transversal en la formación de los futuros periodistas.

En el caso concreto de nuestra experiencia realizada en el aula y para simplificar las numerosas definiciones que giran en torno a la "solidaridad" tomaremos como válida la tesis de Razeto que describe el término de la siguiente forma:

La solidaridad es una relación horizontal entre personas que constituyen un grupo, una asociación o una comunidad, en la cual los participantes se encuentran en condiciones de igualdad. Tal relación o vínculo interpersonal se constituye como solidario en razón de la fuerza o intensidad de la cohesión mutua, que ha de ser mayor al simple reconocimiento de la común pertenencia a una colectividad. Se trata, en la solidaridad, de un vínculo especialmente comprometido, decidido, que permanece en el tiempo y que obliga a los individuos del colectivo que se dice solidario, a responder ante la sociedad y/o ante terceros, cada uno por el grupo, y al grupo por cada uno (Razeto, 2005).

Este valor debe ser promovido a lo largo de todo el proceso formativo de los futuros profesionales de las sociedades modernas si queremos contribuir a una ciudadanía comprometida con el principio de justicia social. En esta tarea, la Universidad tiene una gran responsabilidad como institución última en este proceso, y las prácticas docentes impregnadas de contenido solidario se presentan como un acicate para el logro de este objetivo.

Si partimos de la premisa de la capacidad de incidencia de los medios de información en la Sociedad de la Información y del Conocimiento, el trabajo en el ámbito de la solidaridad de las próximas generaciones de periodistas se plantea como un factor esencial para construir sociedades tolerantes, críticas, y diversas.

En el campo de la comunicación se requiere de periodistas que contribuyan a interpretar, valorar, cuestionar y generar nuevos espacios de diálogo ante los grandes desajustes globales de un modelo societario de consumo e individualista, que repercute en el escenario global, pero también en el local. Tal como mantiene Manzano y Andrés (2007: 30), del seno de las universidades parten aquellos "cuyo comportamiento posterior ayudará a configurar el mundo, manteniendo las inercias o facilitando nuevas soluciones".

El texto que se presenta aborda la solidaridad y su puesta en escena metodológica en el aula y las impresiones suscitadas en el alumnado con el fin último de crear entornos de debate, reflexión y concienciación de una dimensión tan básica y necesaria como es la solidaridad para la convivencia del ser humano. En concreto, el presente estudio expone la experiencia innovadora realizada con estudiantes de segundo curso de Grado en Periodismo de la Universidad de Málaga (curso 2012/13) así como las claves para que pudiera extrapolarse a otras materias del campo de las 
Ciencias Sociales. Este tipo de acción innovadora no solo contribuirá a la toma de conciencia deontológica y a la mejora de la práctica profesional futura del alumnado implicado, sino que además ha permitido la elaboración de estudios, materiales y recursos, fruto de dicho proyecto como el que a continuación se expone.

\section{Hacia una relación entre solidaridad y competencias académicas}

Vivimos inmersos en la vorágine de la denominada Sociedad de la Información y el Conocimiento lo que supone estar sumergidos en un medio que posee una alta densidad comunicativa, es decir, un medio en el cual circula una gran cantidad de saberes y de información, a una alta velocidad y copando diversos espacios de la cotidianidad de quienes, por supuesto, tienen acceso a este mercado mundial de símbolos Valderrama (2007: 28).

Este contexto produce mayores posibilidades de elección pero también nuevos retos para las propias sociedades y sus sistemas formativos. Debemos ser conscientes de que el poder de los medios de comunicación en esta Sociedad de la Información y el Conocimiento es definitorio a la hora de incidir en los modelos sociales seguidos por los ciudadanos y la solidaridad, así como otros valores para la construcción de ciudadanía, no se promueve suficientemente como valor fundamental dentro de sus contenidos (Ruiz Mora, Olmedo Salar, 2011). De hecho los medios de comunicación son competitivos entre ellos y en su discurso suelen ensalzar valores como el éxito, la competitividad o el individualismo. Además, los medios no recogen las voces de los grupos más débiles tales como minorías étnicas, religiosas, discapacitados o marginados dominando en ellos el discurso institucional y los intereses económicos.

La solidaridad tampoco está presente en los estudios de Periodismo, pues al fin y al cabo el propio sistema educativo no es solidario ni integrador sino competitivo y selectivo.

Si tenemos en cuenta que los estudiantes de periodismo serán los trabajadores y responsables de los medios de comunicación de un futuro próximo, creemos oportuno que dentro de la formación específica que reciben para dominar las técnicas y rutinas de la profesión se incluyan de forma transversal contenidos que les permitan reflexionar en torno a la necesidad de crear una ciudadanía más solidaria, además de otros valores tales como la democracia, el medio ambiente, la igualdad, etc. El periodista debe al fin y al cabo ser

Un profesional con una sólida formación en el conocimiento de un entorno diverso, multicultural y dinámico que le permita ser un agente de cambio a través de los medios de comunicación y en las instituciones que requieren optimizar el manejo de la información. (ANECA, 2005).

En cuanto a las competencias docentes, se empieza a hacer referencia a ellas al producirse la adecuación del Plan Bolonia al sistema educativo universitario español, 
a través del Real Decreto 1393/2007, de 29 de octubre, por el que se establece la Ordenación de las Enseñanzas Universitarias Oficiales en España. Los factores esenciales que promueve son, entre otros, un conjunto de títulos universitarios homogéneos en todo el espacio común, la enseñanza basada en ciclos (grado, máster, doctorado), nuevas metodologías de enseñanza-aprendizaje, un mayor acercamiento entre las organizaciones empresariales y la Universidad y la medición del aprendizaje por competencias (y no exclusivamente por contenidos).

En el ámbito académico existen numerosas definiciones del concepto competencia como la aportada por Barraycoa y Lasaga (2009) quienes extraen algunas conclusiones del estudio de las diferentes enunciaciones que se han realizado del término competencia:

- El concepto de competencia es genérico y engloba los de habilidad y capacidad.

- Entre las competencias se establecen relaciones y asociaciones de tal manera que se complementan, potencian y perfeccionan.

- Algunas competencias presentan dimensiones interpersonales e individuales. Así, por ejemplo, la competencia de orientación al logro puede suponer tanto la capacidad de proponerse objetivos grupales como individuales.

- Cualquier persona puede adquirir y mejorar las competencias en mayor o menor medida, si bien se detecta que algunas personas muestran una predisposición hacia determinadas competencias.

- La competencia no es un fin en sí mismo sino un medio para alcanzar objetivos individuales y organizativos.

- La definición de cada competencia debe ser objetivable y permitir la incorporación de mecanismos tanto de autoevaluación como de evaluación externa.

En definitiva, y de una manera simplificadora, podemos afirmar que las competencias se conceptualizan como la capacidad real del individuo para dominar el conjunto de tareas que configuran una función en concreto.

Por lo tanto, la arquitectura de las programaciones docentes debe dar respuesta a la consecución de las competencias planteadas.

\section{La necesidad de dar a conocer a actores alternativos en el sistema mediático}

Teóricamente, una vez finalizados los estudios de Periodismo, el alumno está capacitado para responder a la demanda del sector informativo-comunicativo, que exige del periodista una formación que le otorgue capacidad analítico-crítica y de reflexión sobre el mundo que le rodea. 
Como se puede observar la reflexión y toma de conciencia de valores como la solidaridad, la igualdad, el respeto, el ecologismo o la democracia no aparecen reflejados en la programación docente dándose por hecho que el alumno los adquirirá por otras vías.

Por su parte, la Agencia Nacional de Evaluación de la Calidad y Acreditación (ANECA), organismo de referencia para el conjunto del sistema universitario español, confeccionó en 2005 el Libro Blanco de Titulación Grado en Comunicación en el cual establece una división entre competencias disciplinares (saber), competencias profesionales (saber hacer), competencias académicas y otras competencias específicas.

Comprobamos gratamente, que en el último lugar, en el breve apartado de "otras competencias específicas" se valora la conciencia igualitaria sobre las personas y los pueblos así como el respeto por los derechos humanos internacionales, el conocimiento de las grandes corrientes culturales o civilizadoras en relación con los valores fundamentales individuales y colectivos (ANECA, 2005).

Consideramos que para la formación de periodistas solidarios es preciso que los estudiantes conozcan otros sistemas de medios alternativos, además del conformado por los grandes grupos de comunicación. De forma que junto al discurso uniforme del main stream, se abra antes sus ojos otras formas de hacer periodismo, otro tipo de medios de comunicación con unos objetivos menos ambiciosos económica y políticamente y unas ambiciones más democráticas y humanas. Se trata de los medios del Tercer Sector, término que se empezó a formular hacia mediados de los años 70 y que agruparía a todas aquellas organizaciones autónomas sin ánimo de lucro que utilizan primordialmente, aunque no exclusivamente, medios simbólicos prosociales como la solidaridad, el intercambio simbólico y mantienen una relación privilegiada de osmosis con las redes sociales primarias (Herrera, 1998: 27).

Como señala Lester M. Salomon (1999) estas entidades tienen hoy una "oportunidad de incuestionable trascendencia", pues pueden "expresar las preocupaciones ciudadanas, exigir responsabilidad de los poderes públicos, promover la comunidad, cubrir necesidades insatisfechas y, en general, mejorar la calidad de vida".

Como indica Ramis (1996: 185), cuanto más pequeño es el ámbito, mejor funciona el tejido social,

En los pequeños pueblos, en los pequeños barrios, la comunicación personal tiene una gran importancia, en ciudades pequeñas, incluso en ciudades medianas que no estén situadas en áreas metropolitanas, los medios de comunicación social conservan un indudable carácter propio, pero en la misma medida en que aumenta la población, las dificultades para una comunicación "propia", es decir, con un grado aceptable de participación, no ya como sujetos, sino como objetos de la comunicación, decrece en una progresión geométrica. 


\section{La apuesta solidaria y el grado en periodismo}

La actividad que se recoge en este documento se enmarca en el Proyecto de Innovación Educativa "Comsolidar", propuesta incentivada por los programas de innovación docente de la Universidad de Málaga. Esta iniciativa educativa partía de un objetivo inicial que consistía en ofrecer la oportunidad a los estudiantes de comunicación de una experiencia pedagógica y vital en tres áreas importantes:

- Profundizar la formación en comunicación desde su enfoque solidario y humano, intentando desactivar la perspectiva comercial como la única posible.

- La posibilidad de tener una primera experiencia profesional.

- Integrar la solidaridad y el trabajo colaborativo en los procesos formativos a través de experiencias profesionales reales en favor de causas sociales, humanitarias y medioambientales.

Consideramos que estos principios podríamos insertarlos en la programación docente de la nueva asignatura denominada "Técnicas del mensaje en radio y televisión" presente en el segundo curso de Grado en Periodismo de la Facultad de Ciencias de la Comunicación de la Universidad de Málaga y realizada a lo largo del curso académico 2012/2013.

La asignatura fue enmarcada en el primero de los ejes, la de contenidos comunes obligatorios y tratamos de equipararla a la propuesta que realiza la ANECA de "Expresión oral y escrita para los medios de información", cuyo contenido mínimo debía lograr la adquisición de una serie de competencias relacionadas con el ejercicio profesional en dichos medios. Además de esas competencias nuestro objetivo fue realizar una serie de actividades que ayudaran al logro de dichas competencias a la vez que transmitieran transversalmente una serie de valores tales como la solidaridad, el respeto al medio ambiente, etc. Para ello se organizaron ruedas de prensa por parte de representantes de asociaciones ecologistas como Greenpeace o se trabajó a través de ejercicios prácticos el concepto de solidaridad. Esta última experiencia es la que pretendemos presentar en este texto. Pero antes de ellos es preciso describir las características del grupo de estudiantes con el que trabajamos.

La asignatura "Técnicas del mensaje en radio y televisión" aglutina a un total de 102 alumnos/as, que se encuentran distribuidos en cinco grupos prácticos, cuyos miembros oscilan entre los 22 y los 28 estudiantes. Las prácticas han tenido dos bloques bien diferenciados, uno dedicado a la radio (introducción, características del mensaje en radio, principios de locución, etc. pautas para crear un informativo, etc.) y otro a la televisión (introducción, características del mensaje en tv, estructuras de un informativo en televisión, etc.). Desde el principio, el fin del practicum ha sido poner en marcha un informativo, tanto en televisión como en radio, aunque la experiencia que aquí presentamos se puso en práctica en el bloque dedicado al medio radiofónico que por sus características propias nos pareció idóneo para esta actividad innovadora. 
La radio se constituye en un medio que permite jugar con la imaginación, con la creatividad, con los espacios y los tiempos a través de los sonidos (música, habla y paisaje sonoro -ruido-). Esto no ha pasado inadvertido con el paso de los años, otorgando a la radio esa capacidad transcendental para comunicar:

El carácter exclusivamente sonoro del medio constituye una ventaja, pues permite una mayor creatividad y libertad en la elección de los contenidos de los programas: a través del lenguaje de la radio podemos recrear en la mente del receptor cualquier situación, desde un viaje sideral hasta un fiesta en el Caribe (Valls, 1992: 68).

Teniendo en cuenta las virtudes de este medio y la necesidad de articular la propuesta de forma creativa, que contribuyese a la inclusión de la solidaridad en el primer contacto de los alumnos con el medio radiofónico en su proceso de formación académica, consideramos oportuno plantear la asociación de sonidos con un concepto del ámbito de la solidaridad del que debían explicar de forma clara y concisa el criterio de su elección y la definición que estimasen más oportuna del mismo. Con la vinculación directa entre texto y sonido se pretendió que los estudiantes se percatasen de las posibilidades sugerentes del elemento sonoro que facilita la radio. De igual forma, se incentivaba que el alumnado realizase una práctica de locución con un texto meditado y libre.

Los objetivos que perseguimos con la puesta en marcha de esta práctica fueron:

- Promover el valor de la solidaridad entre los estudiantes mediante su aproximación al escenario conceptual de dicho ámbito.

- Dar a conocer la dimensionalidad de la radio y su universo sonoro como vehículo de comunicación.

- Fomentar la creatividad, las habilidades de escritura y expresión, y la capacidad de reflexión.

Para evaluar la práctica descrita se realizó una encuesta, que estuvo disponible durante un mes en el campus virtual de la asignatura, a fin de poder recabar las opiniones del alumnado. Esta encuesta se estructuró en tres ejes principales:

1. Reflexionar sobre la solidaridad: conocer si el trabajo sobre los conceptos del universo de la solidaridad ha servido para profundizar y reflexionar acerca del tema (si los estudiantes han prestado una mayor atención a cuestiones solidarias, si se han aproximado a la acción de alguna ONG u otras organizaciones civiles o si han tenido conversaciones con los compañeros/as al respecto).

2. La solidaridad como un valor necesario en la formación periodística (si consideran que los periodistas deben estar formados en valores o si estiman que ser solidario es una cualidad que debe ser intrínseca en el ejercicio profesional).

3. La radio como medio para la solidaridad: si valoran positivamente la aplicación de la radio para la experiencia (grado de satisfacción de la práctica, adecuación de la utilización de los elementos sonoros para trabajar el ámbito de la solidaridad o si consideran que se ha logrado el objetivo para aprender 
las posibilidades que brinda la radio para comunicar a través de los sonidos -música, paisajes sonoros y habla-).

\section{Resultados de la experiencia en el aula}

Los resultados obtenidos evidencian que la apuesta en el aula por valores solidarios tiene incidencia en la integración de los mismos en el imaginario del alumnado, tal como quedará constatado en la siguiente exposición. Es preciso señalar que los resultados que se muestran a continuación corresponden a las respuestas ofrecidas por los 61 alumnos que libremente decidieron realizar la encuesta de los 102 alumnos que componen el total del grupo de la asignatura. En el perfil de los encuestados, se observa una clara predominancia de la participación de mujeres $(62 \%)$ respecto a hombres $(38 \%)$, circunstancia que también respondería a la presencia mayoritaria de mujeres cursando los estudios.

A la pregunta de si trabajar con el concepto de la solidaridad en clase les ha motivado para acercarse a este campo, el 39\% (24 estudiantes) manifiesta que "sí, me ha ayudado a conocer más del campo". Casi con el mismo apoyo, la respuesta de "sí, me ha motivado -la práctica-, pero no me ha aportado mucho más de lo que sabía" obtiene el 38\% (23 estudiantes). Al liderazgo de estas propuesta, le sigue la afirmación "sí, pues hasta ahora no me había interesado" con el 12\% (7 estudiantes), el valor "otros" con el 8\% (5 estudiantes) y cerrando el capítulo con el 3\% (2 estudiantes) "no, pues no me interesan estos temas". Estos datos reflejan que existe un logro de uno de los objetivos previstos como es la focalización y aproximación con agrado al campo de la solidaridad por parte del alumnado.

Además, podemos señalar que existe una concienciación de los estudiantes que están familiarizados con los temas vinculados a la solidaridad y, si bien por un lado, agradecen la posibilidad brindada para aprender, otros la utilizan para fortalecer sus conocimientos.

Los estudiantes participantes consideran, en primer lugar, que la solidaridad es un "valor necesario para aplicarlo en mi entorno". Dicha opción es apoyada por 19 de los 61 encuestados, lo que representa el 31\%. En segunda posición se localiza la entrada "reflexionar sobre el concepto y su aplicación en la vida diaria", secundada por 17 estudiantes (28\%), seguido por las consideraciones "darme cuenta que es un valor que debo tener más en cuenta en mi vida" y "que puede ser añadido a mi labor profesional", ambas con el apoyo de 9 estudiantes, lo que supone en cada uno de los casos el 15\%. Cierran el capítulo la entrada "otros" con el 6\% (4 estudiantes) y "no me ha servido de nada" con el 5\% (3 estudiantes). 
Gráfico 1. Aportaciones de la práctica solidaria
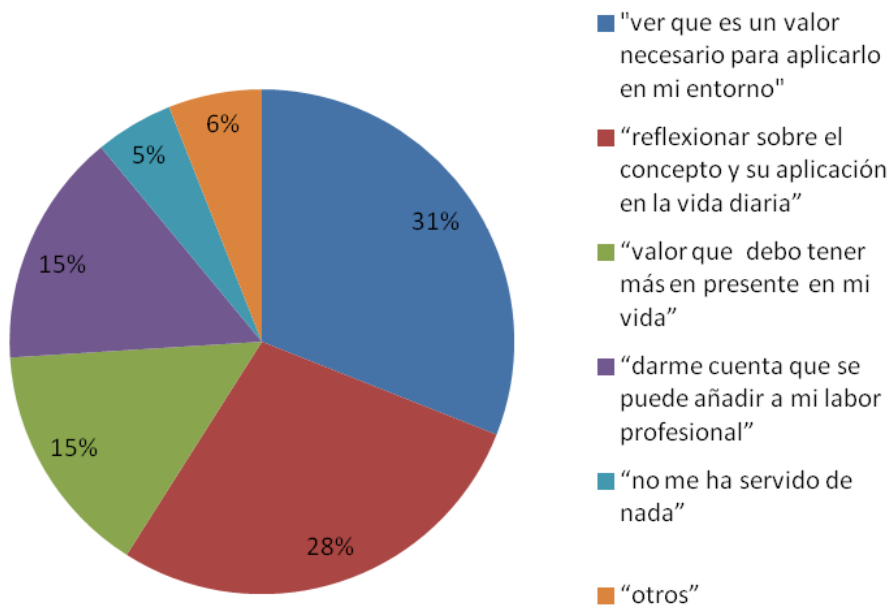

Fuente: Elaboración propia (2013)

Los estudiantes prolongan la incidencia de lo aprendido más allá de su inmediatez del ámbito académico y profesional, entendiendo que el valor de la solidaridad alcanza todas las esferas en las que se ve inmerso el ser humano.

Al objeto de valorar la utilización de la radio y sus recursos se ha preguntado a los estudiantes si creen que la práctica permite apreciar y favorece la capacidad de recreación sonora de la radio. Ante dicha cuestión, el 61\% de los estudiantes, 37 en total, responde de forma afirmativa y sostiene que "permite jugar con distintos elementos sonoros y ver la potencialidad de la radio". Por otra parte, el 34\%, que supone 21 estudiantes, manifiesta que "sí, pero no en su plenitud" y tan solo el 3\%, representado por 2 participantes, secunda el valor "otros". Finalmente, la opción "no aprecio la capacidad de recreación sonora de la radio" solo recibe el apoyo de un estudiante, el $2 \%$. A la pregunta de si tras la experiencia los estudiantes valoran las posibilidades de la radio para recrear espacios y situaciones solidarias a través de los elementos sonoros, el 95\%, que supone 58 de los 61 estudiantes participantes, da una respuesta afirmativa frente al 5\%, 3 estudiantes, que responde negativamente. A aquellos que han respondido afirmativamente a la pregunta anterior, se les pidió que argumentasen sus respuestas. En este sentido, el mayor número de estudiantes, 47 en total $(67 \%)$ "pone en valor las posibilidades que permite recrear situaciones a través de sonidos (música, voz, ruido)". Le sigue con el apoyo de 26 participantes (43\%) la opción "por la capacidad de emocionar con la voz". Un total de 17 estudiantes (28\%) secunda la entrada "por la propia dinámica de la radio, que me gusta" mientras que 13 estudiantes (21\%) apuestan por la afirmación "porque hace que los mensajes sean más cercanos" y, finalmente, 2 participantes (3\%) se adscriben al descriptor "otros". 
Gráfico 2. Argumentaciones que apoyan la aplicación de la radio para la práctica solidaria
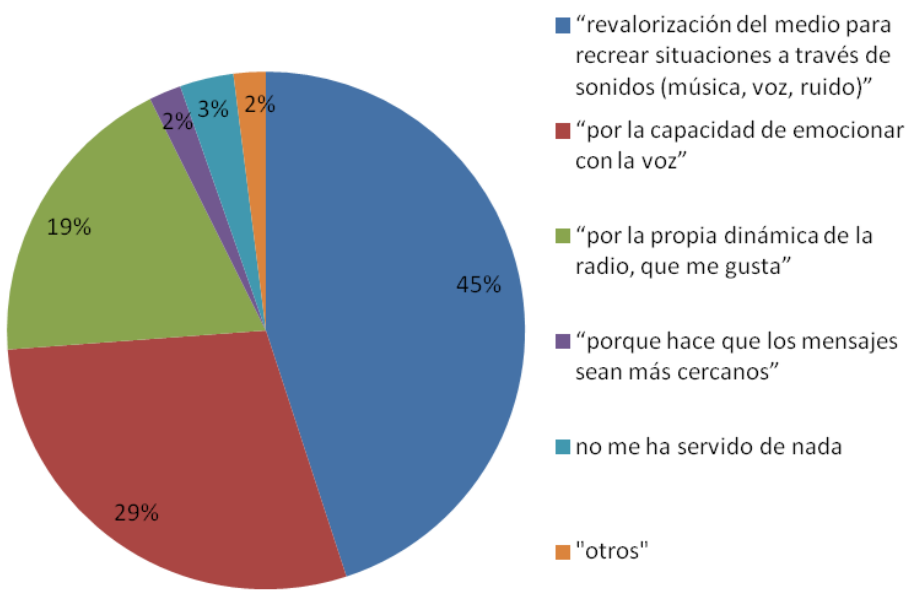

Fuente: Elaboración propia (2013)

Los resultados derivados de las preguntas indicadas previamente ponen en valor el campo radiofónico por su capacidad sugerente y las posibilidades de sus elementos sonoros al servicio del periodista para recrear situaciones.

La situación descrita pone sobre la mesa que la hegemonía de los medios audiovisuales y digitales en nuestros días hace preciso la revalorización de las posibilidades de la radio y su redefinición como medio ágil y cercano, capaz de emocionar, conmover, informar, sugerir y recrear la actualidad de las diferentes voces que componen la realidad.

Sobre la cuestión de si los estudiantes creen que la formación en valores como la solidaridad les servirá en su carrera profesional, resulta significativa la clara postura positiva al respecto. De los 61 alumnos y alumnas, 54 (89\%) consideran que sí les servirá mientras que 7 (11\%) responden que no.

Sin embargo, los datos serán significativamente diferentes cuando los estudiantes contestan acerca del conocimiento sobre la existencia de programas radiofónicos que abordan el campo de la solidaridad. El 82\% (50 estudiantes) manifiesta que no sabe de la existencia de un programa de dichas características y tan solo el 18\% (11 estudiantes) señala que tiene conocimiento al respecto.

Preguntados de forma prospectiva si preferirían trabajar en un medio de comunicación en el que la solidaridad estuviera presente o, al menos, se tratase de forma transversal en sus contenidos, dos opciones obtienen el mismo apoyo: "sí, quisiera trabajar en un medio de comunicación que considerara importante el campo de la solidaridad" y "sí, me gustaría pero no lo veo relevante".

Las propuestas mencionadas obtienen el 39\% del apoyo en cada uno de los casos, lo que significa el respaldo de 24 estudiantes en ambas entradas. 
En segunda posición se sitúa "me daría igual" con el $20 \%$, que representa un total de 12 estudiantes, y con el $2 \%$, con un solo apoyo, el valor "otros".

Gráfico 3. Grado de motivación para trabajar en un medio de comunicación que integre la solidaridad en sus contenidos
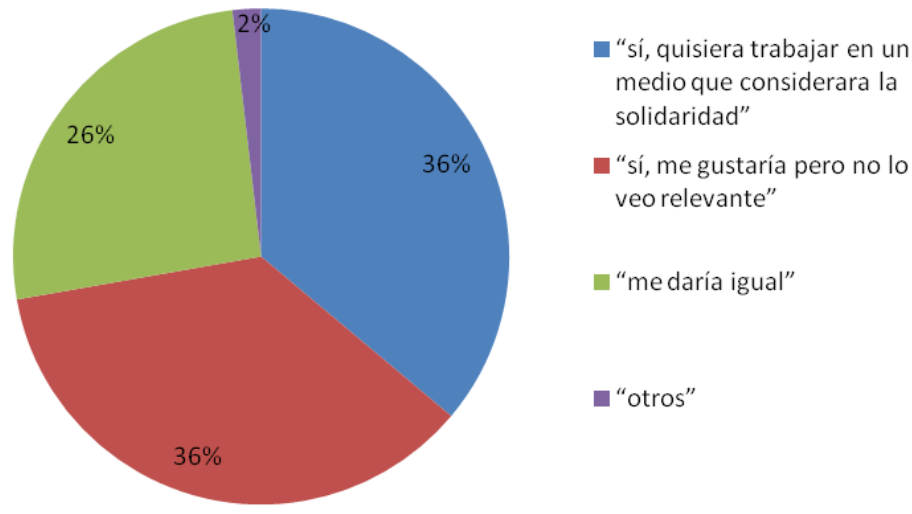

Fuente: Elaboración propia (2013)

Los datos obtenidos sobre la preferencia de trabajar en medios que traten cuestiones de la solidaridad muestra una clara preferencia hacia aquellos que sí lo desean, sin embargo su importancia y relevancia queda mermada al sumar aquellos que no la consideran relevante y "me daría igual".

La práctica de asociar un sonido a un concepto del ámbito de la solidaridad ha generado un amplio abanico de puestas en escena por parte del estudiantado. Algunos de los elementos sonoros que más han destacado los estudiantes van desde el discurso "I have a dream" de Martin Luther King hasta la canción "Imagine" de John Lennon; el bullicio de una taberna; el sonido de la respiración a través de las bombas de oxígeno; el ruido de los trabajos en una construcción; un avisador acústico de semáforo; el sonido de una sartén friendo comida; las señales del telégrafo enviando un mensaje de ayuda o el despegue de un avión con dirección a Eritrea. No obstante, tampoco podían faltar los aplausos, las risas de unos niños, el sonido de mar, de un arpa, el llanto de un bebé o el trinar de los pájaros.

La cualidad más sobresaliente en las prácticas, según los estudiantes, ha sido la originalidad, respaldada por el $31 \%$ del total del alumnado participante (19 estudiantes). A dicha virtud le continúa la capacidad de emocionar con el 25\% (15 estudiantes) y, en tercera posición, la creatividad con el 18\% (11 estudiantes). El descriptor "otros" y "una acertada redacción y locución" cierran este apartado con el 15\% (9 estudiantes) y $11 \%$ (7 estudiantes) respectivamente.

Los resultados permiten vislumbrar que proponer actividades asociadas a valores universales como el caso de la solidaridad promueve un compromiso tácito del grupo, en esta ocasión de los estudiantes. Entendemos que esta motivación ha sido respaldada al mismo tiempo por la libertad con la que las docentes han presentado el tema 
en cuestión, hecho que ha sido valorado positivamente por los alumnos que de forma mayoritaria se han implicado y han decidido participar en la experiencia sin que ello les supusiera recompensa alguna en la calificación final. Esta autonomía también ha servido para la obtención de un variado crisol de trabajos prácticos con amplias dosis de creatividad y originalidad.

Respecto a lo que más han valorado de la práctica, teniendo la posibilidad de elegir dos opciones, "la oportunidad de locutar" ha sido la respuesta que ha registrado el mayor índice de apoyo con el 75\%, seguido de "el compromiso de las docentes por hacernos partícipes de la práctica solidaria" con el 39\%. Por último, "la respuesta de todos mis compañeros/as por implicarse en la práctica" logra el $36 \%$ y "el tema tratado: la solidaridad" registra el $25 \%$.

A modo de conocer el parecer de los estudiantes sobre el funcionamiento de la práctica se les preguntó si consideraban adecuada la organización de la misma. En esta dirección, el 61\% (37 estudiantes) considera que ha sido adecuada, el 36\% muy adecuada (22 estudiantes) y un 3\% (2 estudiantes) regular. Mientras que las entradas poco y nada adecuada no reciben ningún apoyo. Al 96\% (59 estudiantes) le ha gustado la práctica (de los cuales el 44\% manifiesta que "mucho") no registrándose ninguna entrada en el valor de "nada", mientras que "poco" y "regular" solo obtienen un $2 \%$ en cada uno de los casos.

\section{Conclusiones finales}

La realización de esta experiencia nos lleva a determinar que la introducción del valor de la solidaridad debe estar presente en el curriculo formativo de los futuros comunicadores si queremos contribuir a una sociedad impregnada de una cultura de paz.

Las dinámicas basadas en el valor de la solidaridad motivan al alumnado, como hemos podido apreciar en la práctica expuesta, y favorecen puestas en común que alimentan otros imaginarios de construcción de comunidad. Los medios de comunicación requieren profesionales que tengan valores añadidos y que sepan reponder con otros lenguajes, intereses y motivaciones a las nuevas realidades, y que no únicamente respondan al valor del mercadeo de la información.

Es necesario que se abunde en un compromiso colectivo tanto del profesorado como de los estudiantes para abogar por praxis que promuevan la integración en el aula de principios necesarios como profesionales, pero también como ciudadano y ciudadana. Y la solidaridad es un elemento imprescindible para alimentar los lazos sociales y comunicativos tanto en la proximidad como en la globalidad. 


\section{Bibliografía}

ANECA (2005): Libro Blanco. Títulos de grado en Comunicación, Madrid. Agencia Nacional de Evaluación de la Calidad y Acreditación: Madrid.

BARRAYCOA, J. y LASAGA, O. (2009): Competencias e inserción laboral: un análisis de la empleabilidad de los recién licenciados en ADE y Económicas. Barcelona: CEU Ediciones.

CARRILLO I. (Coord.) (2005): Diez valores para el siglo XXI. Barcelona: Cuadernos de Pedagogía.

CONILL J. y GOZÁLVEZ V. (Coord.) (2004): Ética de los medios. Una apuesta por la ciudadanía audiovisual. Barcelona: Gedisa.

COROMINAS Agustín (1999): Modelos y medios de comunicación de masas. Propuestas educativas en educación en valores. Bilbao: Desclée De Brouwer.

DECLARACIÓN DE BOLONIA (1999): Declaración de los Ministros europeos de educación. UE. Consultado el 22 de diciembre de 2010. Disponible en: http:// europe.eu. int/comm/education/policies/educ/bologn a/bologna.pdf

GONZALEZ, L. (1993): "Nuevas relaciones entre educación, trabajo y empleo en la década de los 90", en Revista Iberoamericana de Educación, №2. Consultado el 13 de agosto de 2013. Disponible en: http://www.rieoei.org/ oeivirt/rie02a03.htm

KAPUŚCIŃSKI, R. (2005). Los cínicos no sirven para este oficio. Sobre el buen periodismo. Barcelona. Anagrama.

LATOUCHE, S. (2007): Sobrevivir al desarrollo. Barcelona. Editorial Icaria.

MANZANO, V.; ANDRÉS, L. (2007): El diseño de la nueva Universidad europea. Algunas causas. Algunas consecuencias. Sevilla: Atrapasueños.

MARTÍNEZ, M. E. (2006). "Las competencias en el profesional de la comunicación”. Unirevista. Vol 1, n³. México.

NOS E. y FUENTES M.J. (2006): Medios de comunicación y solidaridad: reflexiones en torno a la (des)articulación social. Castellón de la Plana: Universitat Jaume I.

RAMÍREZ DE LA PISCINA, T. (1999): "Realidad y utopía de la especialización en el periodismo". Consultado el 20 de diciembre de 2010. Disponible en: www. ehu.es/zer/zer6 /13txema.htm.

RAZETO, L. (2005): "El concepto “solidaridad"” en Pensamiento Crítico Latinoamericano. Conceptos Fundamentales, Volumen III. Ediciones Universidad Católica.

REAL, E. (2003): Formación y ejercicio profesional del periodista en la España del siglo XXI dentro del marco de la Unión Europea. Tesis Doctoral. Madrid: Facultad de Ciencias de la Información, Universidad Complutense.

RUIZ MORA, Isabel; RUIZ MUÑOZ, María Jesús y GUERRERO NAVARRO, Daniel (2012): "El desarrollo de competencias profesionales dentro del marco del EEES. El caso de los grados en Comunicación". Estudios sobre el mensaje periodístico. Vol. 18, núm. especial noviembre, págs.: 839-847. Madrid, Servicio de Publicaciones de la Universidad Complutense.

SIERRA SÁNCHEZ, J. (2010): "Competencias profesionales y empleo en el futuro periodista: el caso de los estudiantes de periodismo de la Universidad San Pablo CEU". Revista Icono14. 1 de Julio de 2010, No Año 8, Vol. 2. pp. 156-175. Consultado el 10 de diciembre de 2010. Disponible en: http://www.icono14.net 
UNESCO (1998): "La educación superior en el siglo XXI. Visión y acción”. Consultado el 10 de enero de 2011. Disponible en: http://www.education.unesco.org/ educprog/wche/declaration_spa.htm

VALDERRAMA C.E. (2007): Ciudadanía y Comunicación. Bogotá: Siglo del Hombre Editores.

VALLS, J. M (1992): Educar con y para la radio, $C L \&$ E: Comunicación, lenguaje y educación, $\mathrm{N}^{\circ} 14,67-76$.

YÁNIZ, C. (2008): Las competencias en el currículo universitario: implicaciones para diseñar el aprendizaje y para la formación del profesorado. Red U. Revista de docencia Universitaria, número monográfico 1. Consultado el 8 de enero de 2013. Disponible en: http://www. um.es/ead/Red_U/m1/

\section{Las autoras}

Carmen del Rocío Monedero Morales. En la actualidad ejerce como Profesora Ayudante Doctor en el Departamento de Periodismo de la Universidad de Málaga. Su línea de investigación está focalizada en el campo de la televisión local en Andalucía y en la Televisión Digital Terrestre en el contexto de la Unión Europea, aunque también ha formado y forma parte de equipos investigadores centrados en los medios y la educación o en comunicación y género. Ha realizado varias estancias de investigación en la Universidad Michel de Motaigne de Burdeos y en varias ocasiones ha evaluado artículos para revistas especializadas como ZER Revista de Estudios de la Comunicación (de la Facultad de Ciencias Sociales y de la Comunicación de la Universidad del País Vasco) y Commons, Comunicacion y Ciudadania Digital. Además ha codirigido la tesis doctoral El Ejercicio del Periodismo en Marruecos. Defendida por Brahim Echaabi en la Universidad de Málaga con la calificación de Cum laude.

Silvia Olmedo Salar. Investigadora y docente en el Departamento de Periodismo de la Universidad de Málaga. Su línea de investigación se ha centrado en el análisis de la aplicación de la comunicación en el ámbito de la Cooperación Internacional y el desarrollo, la construcción de nuevos imaginarios desde la comunicación así como el estudio de la radiotelevisión pública y local. Miembro investigador del Proyecto de Excelencia de la Junta de Andalucía TIC 6593 (2011) y del Proyecto del Ministerio de Ciencia e Innovación CSO2011-29195 (2012-2014). Ha realizado estancias de investigación en la Universidad de Stirling (Escocia), en la Universidad de Roskilde (Dinamarca) y en la Cátedra Unesco de Políticas Culturales y Cooperación de la Universidad de Girona (España). En el ámbito de la docencia, trabaja en la perspectiva de la aplicación de metodologías innovadoras en el aula. Ha trabajado como periodista para distintos medios de comunicación de ámbito nacional y local, desarrollando una prolongada experiencia en agencias de información. 